\title{
Clinical and Molecular Diagnostic Evaluation of Systemic Mastocytosis in the South-Eastern Hungarian Population Between 2001-2013 - A Single Centre Experience
}

\author{
Imelda Marton ${ }^{1}$ • László Krenács ${ }^{2}$ • Enikő Bagdi ${ }^{2}$. \\ Annamária Bakos ${ }^{2}$ • Judit Demeter ${ }^{3}$ - Zita Borbényi ${ }^{1}$
}

\begin{abstract}
Systemic mastocytosis (SM) is a rare chronic myeloproliferative neoplasm with only limited epidemiologic data published so far. We aimed to analyze the clinical and molecular diagnostic features, and the prognosis and cumulative incidence of SM cases in a cohort of south-eastern Hungarian patients of 13 year follow up. In the period 2001-2013, 35 consecutive SM cases were diagnosed in our regional centre. Immunophenotype, KIT D816V mutation frequency and clinical characteristics, and the prognosis impact of clinical subtypes were tested and compared with published data. Indolent SM (ISM) was diagnosed in 14 patients, SM with an associated clonal hematologic non-mast cell lineage disease (SM-AHNMD) in 15 patients and aggressive SM (ASM) in 6 patients. The KIT D816V mutation was found in $11 / 14(78 \%)$ of the ISM cases, in $12 / 15$ (80 \%) of the SMAHNMD cases and in 5/6 (83\%) of the ASM cases. The life expectancy of ISM patients was better, whereas the SMAHNMD and ASM groups exhibited a reduced median
\end{abstract}

Electronic supplementary material The online version of this article (doi:10.1007/s12253-015-9948-1) contains supplementary material, which is available to authorized users.

Imelda Marton

imeldamarton@gmail.com

1 2nd Department of Medicine and Cardiology Centre, Medical Faculty, Albert Szent-Györgyi Clinical Centre, University of Szeged, Szeged, Hungary

2 Laboratory of Tumour Pathology and Molecular Diagnostics, Szeged, Hungary

3 1st Department of Internal Medicine, Semmelweis University, Budapest, Hungary survival. The cumulative incidence for 13 year of the SM was $0.27 / 10,000$. We detected lower 13 year cumulative SM incidence than of published epidemiologic data due to in our analyses involved only those patients who had bone marrow biopsy and histopathologically confirmed SM. This clinical overview clearly showed that the clinical characteristics differ between ISM (UP, anaphylaxis and osteoporosis) and SMAHNMD/ASM (cytopenia, eosinophilia and splenomegaly).

Keywords Myeloproliferative neoplasm $\cdot$ Mastocytosis $\cdot$ KIT D816V mutation · Orphan disease

\section{Introduction}

Systemic mastocytosis (SM) is a rare chronic myeloproliferative neoplasm characterized by morphologically and immunophenotypically abnormal mast cell proliferation in single or multiple extracutaneous organs [1-4]. To date only limited epidemiologic data have been published; the prevalence of SM in Central Europe has been estimated to be 0.5$1: 10,000$ [5]. Local regional data have been reported from Groningen on indolent SM (ISM) [6] and population based epidemiological data from Denmark [7]. The recently established centralized ECNM registry currently collects data from ten European countries, with the aim of comparing the incidence and biology of this rare disease in the regions and countries throughout Europe [8].

The 2008 World Health Organization (WHO) classification categorizes SM patients into various subtypes: ISM; SM with an associated clonal hematologic non-mast cell lineage disease (SM-AHNMD) for example acute myeloid leukaemia (AML) or myelodysplastic syndrome (MDS) or chronic myelomonocytic leukemia (CMML); aggressive SM (ASM); 
mast cell leukaemia (MCL); mast cell sarcoma (MCS); and extracutaneous mastocytoma. On the basis of the histopathological findings and organ damage, a definitive variant the smouldering type of SM (SSM) was also introduced in 2007 [9]. The clinical presentation and prognosis in SM patients varies from indolent asymptomatic to an aggressive disease course. The symptoms can vary with the nature of the organ infiltration (e.g. anaemia, thrombocytopenia, malabsorption, hepato- and splenomegaly possibly causing a secondary organ dysfunction) or the mediator-related symptoms, such as cramps, flushes, diarrhoea or pruritus [10].

The diagnosis of SM is based on the presence of minor (such as the abnormal mast cell morphology in bone marrow; the expression of CD25 and/or CD2 in the abnormal mast cells; the presence of the KIT D $816 \mathrm{~V}$ mutation; the elevated serum total tryptase level) and major (the presence of multifocal clusters of morphologically abnormal mast cells in the bone marrow) criteria $[11,4]$. The KIT D816V gain-of-function mutation, a substitution of aspartate (D) to valine $(\mathrm{V})$ at position 816 in the kinase domain, found on exon 17 , can cause autoactivation of the KIT receptor tyrosine kinase [12, 13]. However, this mutation is not specific for mastocytosis: it has also been reported in gastrointestinal stromal tumours, acute myeloid leukaemia and germ cell tumours $[9,14,15]$. The most of adult SM cases exhibit this mutation $[12,16]$.

It has recently been reported that the presence of KIT D816V may be sufficient to cause ISM but not ASM, though it is still questionable whether this mutation alone could be responsible for the diverse clinical presentation of mastocytosis $[17,11]$. Interestingly it has also been established that the presence of the KIT D $816 \mathrm{~V}$ mutation in all haematopoietic lineages and the presence of elevated beta 2 microglobulin levels are associated with a more aggressive form of mastocytosis, and might be identified as possible predictors of the disease progression $[18,19]$.
The KIT D $816 \mathrm{~V}$ mutation can cause resistance to imatinib mesylate $[17,20,18]$. In contrast, the wild-type KIT and several rare KIT mutations such as $\mathrm{F} 522 \mathrm{C}$ and $\mathrm{V} 559 \mathrm{G}$ have been demonstrated to be imatinib-sensitive [21].

The aim of this work was to calculate the 13 year cumulative incidence of the SM in a south-eastern hungarian haematological clinical centre and to survey the immunophenotypes, the KIT D816V mutation frequency, the clinical characteristics and the life expectancy in each subtype and in each case of SM cases which diagnosis were established on bone marrow biopsy in the last 13 years (2001-2013). At the time of this survey, the centre catered for 1,103,463 inhabitants aged 15 years or more from south-eastern Hungary [22].

\section{Patients and Methods}

\section{Patients and Data Collection}

Our centre is a regional centre that caters for the population of three counties in south-eastern Hungary, Southern Great Plain on map (Fig. 1). Our data originated in part from medical database of our centre, sorting with the ICD code (International Classification of Diseases, ICD-10) outpatient database and the inpatient database, and in part from the bone marrow biopsy findings assessed by the tumour pathology laboratory.

C-KIT mutation examinations are made on every bone marrow biopsy sample evaluated at our centre. Only those cases are considered to involve SM which comply strictly with the WHO 2008 criteria (1 major+1 minor or at least 3 minor criteria together). In Hungary, serum tryptase level measurement is currently still not available, but (even without the determined tryptase levels) all of our presented cases meet the WHO criteria of 2008.

Fig. 1 Introduction of the area of our regional centre in Hungary that caters for the population of three counties in south-eastern Hungary (Southern Great Plain)

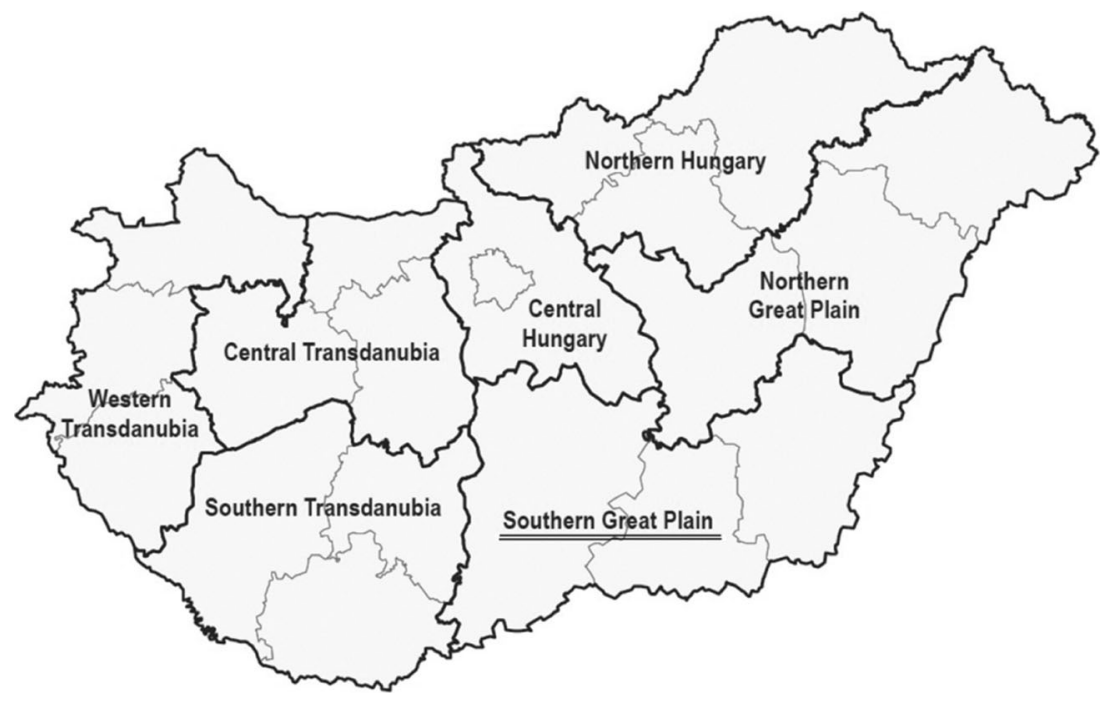


Between 2001 and 2013, 35 patients were diagnosed with SM ( 15 females and 20 males with a median age of 57 years, range: $31-85$ years): 14 with ISM, 15 with SM-AHNMD and 6 with ASM subtypes. All the haematological and pathological results on these patients were collected from the medical data files and reviewed with the approval of the Regional and Institutional Human Medical Biological Research Ethics Committee. Informed consent was not required. The study was conducted in accordance with the Declaration of Helsinki. The haematological management strategy was based on the current available recommendations at the time of the patient's diagnosis, and the therapy initiation $[9,23,24,11$, $25,17,26-28]$.

\section{Pathology}

All bone marrow trephines were routinely fixed in Schaffer's fixative, decalcified in $12.5 \%(w / v) \mathrm{pH} 7.0$ EDTA solution, and embedded into paraffin. Diagnostic immunohistochemical stains to CD117, CD25, mast cell tryptase, CD68, and phospho-STAT5 were made following a heat-induced antigen retrieval.

To detect KIT D816V mutation, polymerase chain reaction (PCR) was performed on each bone marrow trephine utilizing an allele-specific method (Schumacher) with modified parameters [29]. The results were validated by direct sequencing using an Applied Biosystems ${ }^{\circledR} 3500$ DX Series genetic analyzer [29].

\section{Statistical Analysis}

Kaplan-Meier statistical analyses were made to evaluate the survival probability in the various SM subgroups (ISM, SMAHNMD, and ASM) .

For each year we calculated the incidence rate as the number of new cases divided by the mid-year population size. The latter was calculated as the mean of the population size on 1st January on the relevant year and the next year. The cumulative incidence

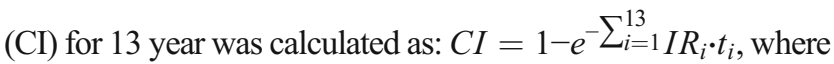
$I R_{i}$ denotes the yearly incidence rates from the $1^{\text {st }}$ to the $13^{\text {th }}$ year, and $t_{i}$ denotes the length of each time period, which is 1 year in this case for all the 13 periods.

\section{Results}

The bone marrow biopsies revealed ISM in 14 patients $(8$ males and 6 females) with a median age of 55 years (range: 31-81 years). The median follow-up time was 50.5 months (range: 5-240 months). Cutaneous lesions (urticaria pigmentosa) were detected in $57 \%(8 / 14)$ of the ISM patients. Mediator-related symptoms appeared in $28 \%(4 / 14)$ of them. The KIT D816V mutation was detected in $78 \%(11 / 14)$. The main characteristics are introduced at Tables 1 and 2, while the detailed data on the clinical symptoms, the immunophenotypes, PCR and sequencing analysis in each patient are introduced in Supplementum 1 and 2.

SM-AHNMD was diagnosed in 15 patients $(7$ males and 8 females) with a median age of 57 years (range: 34-72 years). The median duration of follow-up was 25 months (range: 1104 months). The bone marrow biopsy was indicated in these cases by the symptoms of the associated neoplasm, such as a bone lesion, or clinically relevant blood cell count abnormalities, for example eosinophilia or the elevated or decreased platelet or white blood cell count. This subtype of SM was associated with myelodysplastic syndrome (MDS) or with AML in 3 cases each, with myelofibrosis (MF) in 2 cases, or with ET, PV, chronic myeloid leukaemia (CML), hypereosinophilic syndrome (HES), multiple myeloma (MM), peripheral T-cell lymphoma (PTCL) or B-cell acute lymphoblastic leukaemia (B-ALL) in 1 case each. No cutaneous lesions or mediator-related symptoms were detected in these cases. The KIT D816V mutation was detected in $80 \%$ $(12 / 15)$. The detailed data on the individual SM-AHNMD patients are presented in Supplementum 1 and 2.

ASM was diagnosed in 6 patients (4 males and 2 females) with a median age of 65 years (range: $54-85$ years). The median follow-up period was 20.5 months (range: $2-$ 35 months). The indications of bone marrow biopsy in these cases were hepatosplenomegaly with or without pancytopenia/anaemia/eosinophilia and a weight loss. According to the WHO criteria at least one or more C findings (marked cytopenia; osteolysis with or without pathologic fractures; ascites and elevated liver enzymes; malabsorption plus hypalbuminaemia, palpable splenomegaly with hypersplenism) were present in all of ASM cases $[23,11]$. Cutaneous lesions were observed in $33 \%$ $(2 / 6)$ of the patients. Mediator-related symptoms were present in 1 case. The KIT D $816 \mathrm{~V}$ mutation was detected in $83 \%(5 / 6)$ of the patients. The detailed data are to be seen in Supplementum 1 and 2.

The survival data of each SM subtype according to KaplanMeier survival curve analysis are presented in Fig. 2.

The median survival for the ASM group was 1.73 years, while that for the SM-AHNMD group did not reach the median during the observed follow-up period. No patients died during the follow-up period in the ISM group. The MantelCox, Breslow and Tarone-Ware tests uniformly led to $p=$ 0.000 , demonstrating that the survival differed significantly in the observed subgroups.

Our regional medical university centre receives SM patients from among 1,103,463 inhabitants aged 15 years or more in south-eastern Hungary [22], and this allowed to calculate the cumulative incidence for 13 year of the of SM in general population, which proved to be $0.27 / 10,000$ in this region. 
Table 1 Main clinical characteristics of the patients

\begin{tabular}{|c|c|c|c|}
\hline Characteristics & ISM & SM-AHNMD & ASM \\
\hline Patients (N) & 14 & 15 & 6 \\
\hline Males (N) & 8 & 7 & 4 \\
\hline Females $(\mathrm{N})$ & 6 & 8 & 2 \\
\hline Median age at diagnosis, median (years) (range) & $55(31-81)$ & $57(34-72)$ & $65(54-85)$ \\
\hline Median follow up (months) (range) & $50.5(5-240)$ & $25(1-104)$ & $20.5(2-35)$ \\
\hline Associated haematological disease & none & $\begin{array}{l}\text { MDS(3);AML(3);MF (2); ET(1); PV(1);CML(1); } \\
\text { iHES(1);MM(1);PTCL(1); B-ALL(1) }\end{array}$ & none \\
\hline Urticaria pigmentosa & $8 / 14$ & none & none \\
\hline $\begin{array}{l}\text { Mediator-related symptoms: } \\
\text { skin }(\mathrm{S}) \\
\text { gastrointestinal }(\mathrm{GI}) \\
\text { cardiovascular }(\mathrm{CV}) \\
\text { neurological }(\mathrm{N}) \\
\text { anaphylaxis }(\mathrm{A})\end{array}$ & $\begin{array}{l}\text { S: } 5 / 14 \\
\text { GI: } 3 / 14 \\
\text { CV: } 2 / 14 \\
\text { N: } 1 / 14 \\
\text { A: } 2 / 14\end{array}$ & none & $\begin{array}{l}\mathrm{S}: 2 / 6 \\
\mathrm{~N}: 1 / 6\end{array}$ \\
\hline $\begin{array}{l}\text { Coexistent allergy } \\
\text { (inhalative, nutritive, drug, insect) }\end{array}$ & $3 / 14$ & none & none \\
\hline Constitutional symptoms & $2 / 14$ & $1 / 15$ & $6 / 6$ \\
\hline $\begin{array}{l}\text { Organ damage / organopathy: } \\
\text { Hepatomegaly }(\mathrm{H}) \text {, Splenomegaly (S), } \\
\text { Adenopathy(A), } \\
\text { Osteopenia/ } \\
\text { osteoporosis/osteolysis(O) }\end{array}$ & $\begin{array}{l}\mathrm{H}: 2 / 14 \\
\mathrm{H}^{*}: 1 / 14 \\
\mathrm{~A}: 1 / 14 \\
\mathrm{O}: 3 / 14\end{array}$ & $\begin{array}{l}\mathrm{H}: 3 / 15 \\
\mathrm{~S}: 2 / 15 \\
\mathrm{~A}: 1 / 15\end{array}$ & $\begin{array}{l}H^{*}: 6 / 6 \\
\text { S: } 3 / 6 \\
\text { A: } 3 / 6 \\
\text { O: } 3 / 6\end{array}$ \\
\hline $\mathrm{C}$ findings & none & none & $6 / 6$ \\
\hline
\end{tabular}

Abbreviations: ASM aggressive systemic mastocytosis; ISM Indolent systemic mastocytosis; SM-AHNMD systemic mastocytosis with an associated clonal hematologic non-mast cell lineage disease;mediator- related symptoms; $S$ skin (flush, pruritus); $G I$ gastrointestinal symptoms (diarrhoea); $C V$ cardiovascular (palpitation, dizziness, syncope); $N$ neurologic; $A$ anaphylaxis; Constitutional symptoms were observed generalized weakness, fatigue, sweats, chills, arthrarlgias, myalgias; Organ damage/ organopathy: hepatomegaly $(\mathrm{H}) \mathrm{H}^{*}$ : hepatomegalia with elevated alkaline phosphatase; splenomegaly (S); Adenopathy (A);Osteopenia/osteoporosis/osteolysis (O);iHES: idiopathic hypereosinophilic syndrome; MDS: myelodysplastic syndrome; AML: acute myeloid leukaemia; ET: essential thrombocythaemia; MM: multiple myeloma; MF: myelofibrosis; PTCL: peripheral T-cell lymphoma; CML: chronic myeloid leukaemia; B-ALL: B-cell acute lymphoblastic leukaemia; PV: polycythaemia vera

Four patients (cases 2, 13, 29 and 35) were excluded from this cumulative incidence estimation: although they were diagnosed in our regional centre, they were not inhabitants of this region of Hungary.

\section{Discussion}

The aim of this paper was to summarize our clinical experience in SM in the period 2001-2013. Since there is no official national mastocytosis registry in Hungary, we aimed to use our regional data to calculate the cumulative incidence of SM in a south-eastern hungarian clinical centre, based on only those cases (introduced above) which were referred to histopathological analysis of bone marrow biopsy and the presence of SM were confirmed.

Our analyses demonstrated that the main cause of the indication of the bone marrow biopsy in the group of ISM patients was urticaria pigmentosa $(8 / 14 ; 57 \%)$. It is important, therefore, that the dermatologist should refer the patients to haematological centres to establish the potential bone marrow involvement in the presence of cutaneous mastocytosis lesions. In other cases, the bone marrow biopsy was indicated by eosinophilia $(3 / 14 ; 21 \%)$, anaemia $(1 / 14 ; 7 \%)$, lytic bone lesions $(1 / 14 ; 7 \%)$ or adenomegaly $(1 / 14 ; 7 \%)$.

Table 2 The main characteristics indicating bone marrow biopsy, and the main results

\begin{tabular}{llllll}
\hline Subgroups & Main clinical symptoms /findings/features & KIT mutation (PCR) D816V(+) & BM dysplasia & BM fibrosis & BM eosinophilia \\
\hline ISM & urticaria, anaphylaxis,osteoporosis & $11 / 14$ & $1 / 14$ & $0 / 14$ & $6 / 14$ \\
SM-AHNMD & cytopenias, splenomegaly, & $12 / 15$ & $5 / 15$ & $3 / 15$ & $3 / 15$ \\
ASM & cytopenias, splenomegaly, eosinophilia & $5 / 6$ & $1 / 6$ & $0 / 6$ & $3 / 6$ \\
\hline
\end{tabular}

Abbreviations: ASM aggressive systemic mastocytosis; BM bone marrow; ISM: Indolent systemic mastocytosis; SM-AHNMD systemic mastocytosis with an associated clonal hematologic non-mast cell lineage disease 
Fig. 2 Survival of systemic mastocytosis patients classified by disease type. The observed Kaplan-Meier survival for systemic mastocytosis patients classified by disease type: ISM (blue), SM-AHNMD (green), and ASM (yellow)

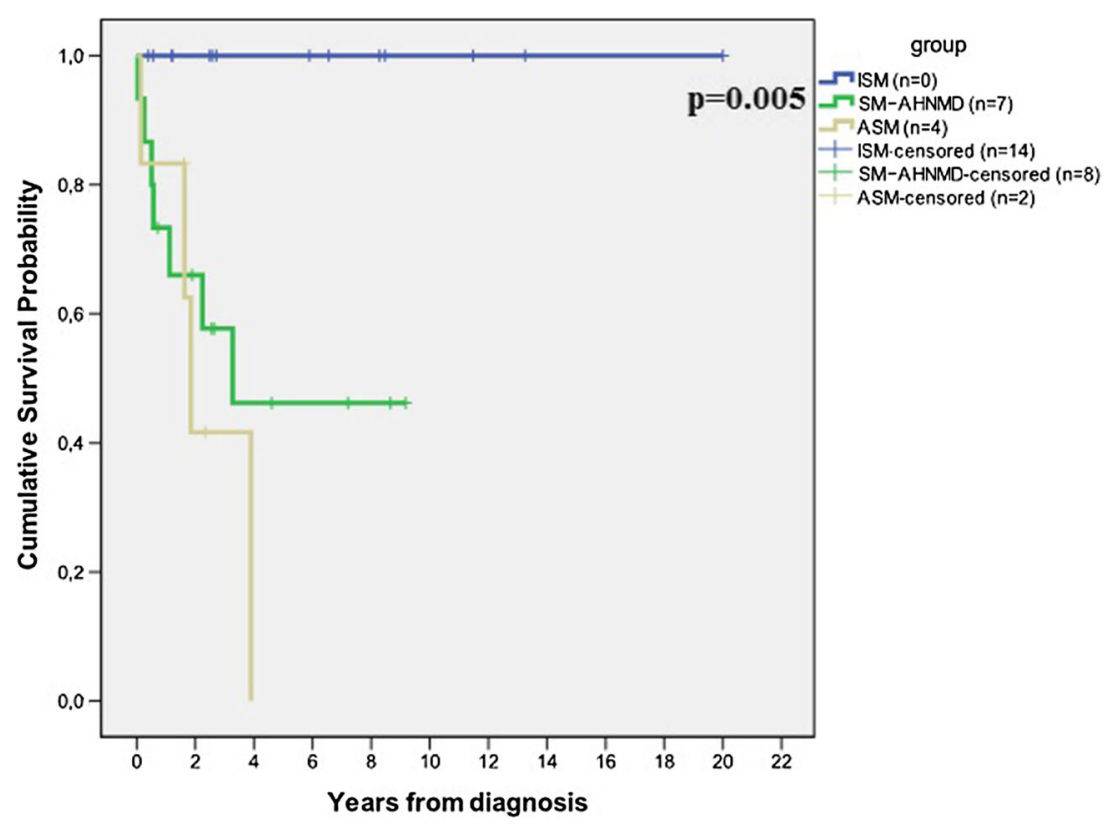

In the SM-AHNMD cases, it was predominantly the symptoms of the associated neoplasm that indicated the bone marrow biopsy. In the majority of our patients (14/15; $93 \%)$, these were symptoms of pancytopenia, anaemia, leukocytosis, thrombocytosis, bone pain/lesion or hepatosplenomegaly. In one case, attention was drawn to the associated SM by the lymphoma staging process. Our finding was consistent with previous reports [16] whereas the most common associated clonal diseases was myeloid neoplasms (12/15 cases; $80 \%$ ) and the associated lymphoid neoplasms was much less common (3/15 cases; $20 \%)$.

In the ASM subgroup, the bone marrow biopsy was indicated in all cases $(6 / 6 ; 100 \%)$ by anaemia with or without thrombocytopenia or hepatosplenomegaly with or without constitutional symptoms.

It has been reported [9] that ISM is mostly associated with cutaneous symptoms and this is in agreement with our own findings ( $8 / 14$ cases; $57 \%$ ), whereas we observed a lower rate of these associations in ASM $(2 / 6 ; 33 \%)$, and there were no such cases in SM-AHNMD.

Only the KIT D816V mutation was detected among our cases, with a frequency close to the reported data [30]. This mutation was positive in $78 \%(11 / 14)$ of the ISM cases, $80 \%$ $(12 / 15)$ of the SM-AHNMD cases, and $83 \%(5 / 6)$ of the ASM patients.

Lim et al. concluded that the life expectancy in ISM was superior to that in non-ISM, and not significantly different from that for the age- and sex-matched US population, a result that validated the prognostic relevance of the WHO subclassification of SM [16]. A long-term study of the Spanish Network on Mastocytosis also led to the finding that the great majority of adult ISM patients have a normal life expectancy
[19]. Our analyses of Hungarian patients revealed that the survival rate of SM patients is less than that expected for the age- and sex-matched Hungarian population; The life expectancy of the patients with ISM was excellent, whereas the SMAHNMD and ASM groups exhibited a reduced median survival. This distribution of subtypes and survival pattern in our study was similar to previously published in the Mayo clinic study [17].

The recently established centralized ECNM registry currently collects data from ten European countries, and epidemiological data are already therefore available as concerns the prevalence of SM [8]. The prevalence of mastocytosis in Central Europe has been estimated to be $\sim 0.5-1 / 10,000$ [5]. Our findings of the 13-year cumulative incidence of SM in general population aged 15 years or more was $0.27 / 10,000$ fit the incidence of orphan diseases. As in the most relevant literature, we can not report a gender predominance [5].

\section{Limitations}

Since only cases that complied strictly with the WHO criteria were included as SM cases, the cumulative incidence of SM calculated during our research is likely to be somewhat underestimated. On this basis, the 13-year cumulative incidence of SM in our research was $0.27 / 10,000$, and only the clinical features of this group of patients were considered.

Only the SM cases were enrolled in our detailed investigation. Those patients with skin lesions, who refused the BM biopsy, were not enrolled in our calculations.

The cumulative incidence would be increased by inclusion of the patients (3) with only two minor criteria (whose data are not included in the present manuscript), whose serum tryptase 
examinations may later lead to some of them fulfilling the three minor WHO criteria.

The real cumulative incidence might be higher because SM is often underdiagnosed and the symptoms can be subtle or absent.

\section{Conclusion}

This clinical overview clearly showed that the clinical characteristics differ between ISM (UP, anaphylaxis and osteoporosis) and SM-AHNMD/ASM (cytopenia, eosinophilia and splenomegaly).

Despite the existence of the centralized ECNM registry and the avaliability of some epidemiological data on the incidence and prevalence of SM, the lack of an official national mastocytosis registry in Hungary led us to apply our regional data to calculate the cumulative incidence of SM in southeastern Hungary. This cumulative incidence of SM in adults was lower compared to the epidemiologic data reported in the literature due to in our analyses involved only those patients who had bone marrow biopsy and histopathologically confirmed SM [6].

Acknowledgment The authors would like to acknowledge Balázs Kotosz $\mathrm{PhD}$ for his help with statistical analysis and Professor Zoltán Vokó MD. PhD. DSc for epidemiological supervision.

Competing interests The authors declare that they have no competing interests.

\section{References}

1. Brunning RD, McKenna RW, Rosai J, Parkin JL, Risdall R (1983) Systemic mastocytosis. Extracutaneous manifestations. Am J Surg Pathol 7(5):425-438

2. Stevens EC, Rosenthal NS (2001) Bone marrow mast cell morphologic features and hematopoietic dyspoiesis in systemic mast cell disease. Am J Clin Pathol 116(2):177-182. doi:10.1309/Q2WJ46CL-YRFT-M5JF

3. Gotlib J, Pardanani A, Akin C, Reiter A, George T, Hermine O, Kluin-Nelemans H, Hartmann K, Sperr WR, Brockow K, Schwartz LB, Orfao A, Deangelo DJ, Arock M, Sotlar K, Horny HP, Metcalfe DD, Escribano L, Verstovsek S, Tefferi A, Valent P (2013) International Working Group-Myeloproliferative Neoplasms Research and Treatment (IWG-MRT) \& European Competence Network on Mastocytosis (ECNM) consensus response criteria in advanced systemic mastocytosis. Blood 121(13):2393-2401. doi: 10.1182/blood-2012-09-458521

4. Wadleigh M, Tefferi A (2010) Classification and diagnosis of myeloproliferative neoplasms according to the 2008 World Health Organization criteria. Int J Hematol 91(2):174-179. doi:10.1007/ s12185-010-0529-5

5. Valent P (2013) Mastocytosis: a paradigmatic example of a rare disease with complex biology and pathology. Am J Cancer Res 3(2):159-172
6. van Doormaal JJ, Arends S, Brunekreeft KL, van der Wal VB, Sietsma J, van Voorst Vader PC, Oude Elberink JN, KluinNelemans JC, van der Veer E, de Monchy JG (2013) Prevalence of indolent systemic mastocytosis in a Dutch region. J Allergy Clin Immunol 131(5):1429-1431 e1421. doi:10.1016/j.jaci.2012.10. 015

7. Cohen SS, Skovbo S, Vestergaard H, Kristensen T, Moller M, Bindslev-Jensen C, Fryzek JP, Broesby-Olsen S (2014) Epidemiology of systemic mastocytosis in Denmark. Br J Haematol. doi:10.1111/bjh.12916

8. Valent P, Arock M, Bonadonna P, Brockow K, Broesby-Olsen S, Escribano L, Gleixner KV, Grattan C, Hadzijusufovic E, Hagglund H, Hermine O, Horny HP, Kluin-Nelemans HC, Maurer M, Niedoszytko M, Nedoszytko B, Nilsson G, Oude-Elberink HN, Orfao A, Radia D, Reiter A, Siebenhaar F, Sotlar K, Sperr WR, Triggiani M, VanDoormaal JJ, Varkonyi J, Yavuz S, Hartmann K (2012) European Competence Network on Mastocytosis (ECNM): 10 -year jubilee, update, and future perspectives. Wien Klin Wochenschr 124(23-24):807-814. doi:10.1007/s00508-012-0293-Z

9. Valent P, Akin C, Escribano L, Fodinger M, Hartmann K, Brockow K, Castells M, Sperr WR, Kluin-Nelemans HC, Hamdy NA, Lortholary O, Robyn J, van Doormaal J, Sotlar K, Hauswirth AW, Arock M, Hermine O, Hellmann A, Triggiani M, Niedoszytko M, Schwartz LB, Orfao A, Horny HP, Metcalfe DD (2007) Standards and standardization in mastocytosis: consensus statements on diagnostics, treatment recommendations and response criteria. Eur J Clin Invest 37(6):435-453. doi:10.1111/j.1365-2362.2007.01807.x

10. Valent P, Akin C, Arock M, Brockow K, Butterfield JH, Carter MC, Castells M, Escribano L, Hartmann K, Lieberman P, Nedoszytko B, Orfao A, Schwartz LB, Sotlar K, Sperr WR, Triggiani M, Valenta R, Horny HP, Metcalfe DD (2012) Definitions, criteria and global classification of mast cell disorders with special reference to mast cell activation syndromes: a consensus proposal. Int Arch Allergy Immunol 157(3):215-225. doi:10.1159/000328760

11. Pardanani A (2013) Systemic mastocytosis in adults: 2013 update on diagnosis, risk stratification, and management. Am J Hematol. doi:10.1002/ajh.23459

12. Garcia-Montero AC, Jara-Acevedo M, Teodosio C, Sanchez ML, Nunez R, Prados A, Aldanondo I, Sanchez L, Dominguez M, Botana LM, Sanchez-Jimenez F, Sotlar K, Almeida J, Escribano L, Orfao A (2006) KIT mutation in mast cells and other bone marrow hematopoietic cell lineages in systemic mast cell disorders: a prospective study of the Spanish Network on Mastocytosis (REMA) in a series of 113 patients. Blood 108(7):2366-2372. doi:10.1182/blood-2006-04-015545

13. Soucie E, Hanssens K, Mercher T, Georgin-Lavialle S, Damaj G, Livideanu C, Chandesris MO, Acin Y, Letard S, de Sepulveda P, Hermine O, Bernard OA, Dubreuil P (2012) In aggressive forms of mastocytosis, TET2 loss cooperates with c-KITD816V to transform mast cells. Blood 120(24):4846-4849. doi:10.1182/blood-2011-12397588

14. Tay CM, Ong CW, Lee VK, Pang B (2013) KIT gene mutation analysis in solid tumours: biology, clincial applications and trends in diagnostic reporting. Pathology 45(2):127-137. doi:10.1097/ PAT.0b013e32835c7645

15. Beghini A, Peterlongo P, Ripamonti CB, Larizza L, Cairoli R, Morra E, Mecucci C (2000) C-kit mutations in core binding factor leukemias. Blood 95(2):726-727

16. Lim KH, Tefferi A, Lasho TL, Finke C, Patnaik M, Butterfield JH, McClure RF, Li CY, Pardanani A (2009) Systemic mastocytosis in 342 consecutive adults: survival studies and prognostic factors. Blood 113(23):5727-5736. doi:10.1182/blood-2009-02-205237

17. Pardanani A, Tefferi A (2010) Systemic mastocytosis in adults: a review on prognosis and treatment based on 342 Mayo Clinic patients and current literature. Curr Opin Hematol 17(2):125-132. doi:10.1097/MOH.0b013e3283366c59 
18. Verstovsek S (2013) Advanced systemic mastocytosis: the impact of KIT mutations in diagnosis, treatment, and progression. Eur $\mathrm{J}$ Haematol 90(2):89-98. doi:10.1111/ejh.12043

19. Escribano L, Alvarez-Twose I, Sanchez-Munoz L, Garcia-Montero A, Nunez R, Almeida J, Jara-Acevedo M, Teodosio C, GarciaCosio M, Bellas C, Orfao A (2009) Prognosis in adult indolent systemic mastocytosis: a long-term study of the Spanish Network on Mastocytosis in a series of 145 patients. J Allergy Clin Immunol 124(3):514-521. doi:10.1016/j.jaci.2009.05.003

20. Ustun C, DeRemer DL, Akin C (2011) Tyrosine kinase inhibitors in the treatment of systemic mastocytosis. Leuk Res 35(9):11431152. doi:10.1016/j.leukres.2011.05.006

21. Akin C, Fumo G, Yavuz AS, Lipsky PE, Neckers L, Metcalfe DD (2004) A novel form of mastocytosis associated with a transmembrane c-kit mutation and response to imatinib. Blood 103(8):32223225. doi:10.1182/blood-2003-11-3816

22. Hungarian Central Statistical Office-Population census (2011). Regional data-Bács-Kiskun county http://www.ksh.hu/ nepszamlalas/tables_regional_03; Regional data- Békés county http://www.ksh.hu/nepszamlalas/tables_regional_04; Regional data - Csongrád county http://www.ksh.hu/nepszamlalas/tables regional_06

23. Valent P, Sperr WR, Akin C (2010) How I treat patients with advanced systemic mastocytosis. Blood 116(26):5812-5817. doi:10. 1182/blood-2010-08-292144
24. Lim KH, Pardanani A, Butterfield JH, Li CY, Tefferi A (2009) Cytoreductive therapy in 108 adults with systemic mastocytosis: Outcome analysis and response prediction during treatment with interferon-alpha, hydroxyurea, imatinib mesylate or 2chlorodeoxyadenosine. Am J Hematol 84(12):790-794. doi:10. 1002/ajh.21561

25. Pardanani A (2013) How I treat patients with indolent and smoldering mastocytosis (rare conditions but difficult to manage). Blood 121(16):3085-3094. doi:10.1182/blood-2013-01-453183

26. Valent P (1996) Biology, classification and treatment of human mastocytosis. Wien Klin Wochenschr 108(13):385-397

27. Escribano L, Akin C, Castells M, Orfao A, Metcalfe DD (2002) Mastocytosis: current concepts in diagnosis and treatment. Ann Hematol 81(12):677-690. doi:10.1007/s00277-002-0575-Z

28. Valent P, Akin C, Sperr WR, Horny HP, Arock M, Lechner K, Bennett JM, Metcalfe DD (2003) Diagnosis and treatment of systemic mastocytosis: state of the art. Br J Haematol 122(5):695-717

29. Schumacher JA, Elenitoba-Johnson KS, Lim MS (2008) Detection of the c-kit D816V mutation in systemic mastocytosis by allelespecific PCR. J Clin Pathol 61(1):109-114. doi:10.1136/jcp.2007. 047928

30. Akin C (2005) Clonality and molecular pathogenesis of mastocytosis. Acta Haematol 114(1):61-69. doi:10.1159/ 000085563 